\title{
Ualidação psicométrica da Escala da Relação Coparental em mães portuguesas
}

\author{
Psychometric Ualidation of the Coparenting Relationship Scale \\ in Portuguese mothers \\ Ualidación psicométrica de la Escala de la Relación Coparental \\ en madres portuguesas
}

\author{
Diogo Lamela* \\ Ana Morais \\ Inês Jongenelen* \\ Universidade Lusófona do Porto, Porto, Portugal.
}

Doi: http://dx.doi.org/10.12804/revistas.urosario.edu.co/apl/a.5564

\section{Resumo}

O presente estudo teve como objetivo a adaptação e a validação da Escala de Relação Coparental —ERC — em mães portuguesas. A versão original da medida avalia as quatro dimensões da coparentalidade do modelo ecológico da coparentalidade, através de 7 subescalas. O estudo foi composto por uma amostra de 548 mães numa relação íntima heterossexual, e pelo menos um filho com o atual parceiro conjugal com uma idade inferior a 18 anos. Testada através de análise fatorial confirmatória, a solução fatorial final investigada apresentou um ajustamento satisfatório, o que sugere a validade de construto da ERC, $\chi^{2}(372)=828.5, \mathrm{~ns}, \mathrm{CFI}=0.95$, NFI $=0.91$ e RMSEA $=0.04(90 \%$ IC $=0.04-0.05)$. A escala também apresentou coeficientes de consistência interna entre o satisfatório e o elevado em todas as subescalas
( $\alpha$ de Cronbach entre 0.70 e 0.94 ) e valores muito satisfatórios de validade convergente e divergente, obtidos através de análises correlacionais da ERC com outros construtos teoricamente selecionados. Os resultados dão suporte inicial à qualidade psicométrica da versão portuguesa da ERC.

Palavras-chave: coparentalidade, mães, validação, avaliação.

\section{fibstract}

The aim of the present study was to adapt and validate the Coparenting Relationship Scale - CRS - in a sample of Portuguese mothers. The original version of this measure assesses in seven subscales the four dimensions of coparenting described in the ecological model of coparenting. The study was composed by a sample of

* Diogo Lamela, Ana Morais, Inês Jongenelen, Universidade Lusófona do Porto, Porto, Portugal.

Correspondência a respeito deste artigo deve ser endereçada para Diogo Lamela, Universidade Lusófona do Porto. Correio eletrônico: lamela@ulp.pt

Cómo citar este artículo: Lamela, D., Morais, A. \& Jongenelen, I. (2018). Validação Psicométrica da Escala da Relação Coparental em Mães Portuguesas. Avances en Psicología Latinoamericana, 36(3), 585-600. DOI: http://dx.doi.org/10.12804/ revistas.urosario.edu.co/apl/a.5564 
548 mothers having a heterosexual intimate relationship and with at least one child (under 18 years) from the current marital relationship. Using a confirmatory factor analysis, the final factor solution revealed a satisfactory adjustment, suggesting the construct validity of the CRS, $\chi^{2}(372)=828.5, \mathrm{~ns}, \mathrm{CFI}=0.95, \mathrm{NFI}=0.91$ e RMSEA $=0.04(90 \%$ IC $=0.04-0.05)$. The scale also showed coefficients of internal consistency between satisfactory and elevated in all subscales (Cronbach's $\alpha$ between 0.70 and 0.94 ) and satisfactory values of convergent and divergent validity, obtained through correlational analysis of CRS with other theoretically selected constructs. The results give initial support to the psychometric quality of the Portuguese version of CRS. Keywords: Coparenting, mothers, validation, assessment.

\section{Resumen}

El presente estudio tuvo como objetivo la adaptación y la validación de la Escala de Relación Coparental —ERC - en madres portuguesas. La versión original de la medida evalúa las cuatro dimensiones de la coparentalidad del modelo ecológico de la coparentalidad, a través de 7 subescalas. El estudio estuvo compuesto por una muestra de 548 madres en una relación íntima heterosexual y por lo menos un hijo con el actual compañero conyugal, con una edad inferior a 18 años. Probada a través de análisis factorial confirmatorio, la solución factorial final investigada presentó un ajuste satisfactorio, lo que sugiere la validez de constructo de la ERC, $\chi^{2}(372)=828.5, n s, \mathrm{CFI}=0.95, \mathrm{NFI}=0.91 \mathrm{y}$ RMSEA $=0.04(90 \%$ IC $=0.04-0.05)$. La escala también presentó coeficientes de consistencia interna entre lo satisfactorio y lo elevado en todas las subescalas ( $\alpha$ de Cronbach entre 0.70 e 0.94 ) y valores muy satisfactorios de validez convergente y divergente, obtenidos a través de análisis correlacionales de la ERC con otros constructos teóricamente seleccionados. Los resultados dan soporte inicial a la calidad psicométrica de la versión portuguesa de la ERC.

Palabras clave: coparentalidad, madres, validación, evaluación.
Na última década, a investigação tem sugerido que a coparentalidade é um processo familiar com poder preditivo do ajustamento psicológico das crianças, dos pais, da parentalidade e do funcionamento da família (Fagan, Levine, Kaufman \& Hammar, 2016; Feinberg, Brown \& Kan, 2012; Kuersten-Hogan, 2017; Lamela, Jongenelen, Morais \& Figueiredo, 2017). Apesar de ainda não haver um consenso na definição das dimensões que integram o construto, a coparentalidade pode ser globalmente definida pela relação diádica na cooperação e reciprocidade, por parte dos pais na educação, formação e decisões, acerca da vida da criança (Feinberg, 2003; Lamela, Nunes-Costa \& Figueiredo, 2010).

A coparentalidade pode também explicar as interligações entre as relações conjugais, práticas parentais e os níveis de adaptação dos filhos, sendo assim um mediador entre a qualidade da relação conjugal e os percursos desenvolvimentais da criança (Bronte-Tinkew, Horowitz \& Carrano, 2010; Delvecchio, Sciandra, Finos, Mazzeschi \& Riso, 2015; Pedro, Ribeiro \& Shelton, 2012). Por sua vez, a relação coparental é criada quando os cuidadores primários da criança tomam a responsabilidade de cuidar e zelar pelo seu bem-estar (Van Egeren \& Hawkins, 2004; Feinberg, 2003).

Contudo, na perspetiva dos sistemas familiares, os subsistemas conjugal e coparental, apesar de interdependentes, são estruturalmente distintos (Feinberg, 2003; Lamela et al., 2010; McHale \& Lindahl, 2011). Mais concretamente, o subsistema coparental é operacionalizado como um subsistema autónomo, com mecanismos e características idiossincráticas e diferenciáveis dos processos relacionais dos subsistemas conjugal e parental, apresentando uma dimensão sistémica e triádica (pai-mãe-filho) (Favez, Tissot \& Frascarolo, 2016; Korja et al., 2016; Murphy, Jacobvitz \& Hazen, 2016).

Modelos teóricos têm sido desenvolvidos para explicar o funcionamento da coparentalidade de forma a descrever e filtrar as suas componentes 
estruturais, bem como a sua função no seio familiar (Belsky, Crnic \& Gable, 1995; Van Egeren \& Hawkins, 2004; Margolin, Gordis \& John, 2001; McHale, 1995, 1997). Numa das propostas iniciais sobre as componentes definidoras do construto, Margolin et al. (2001) sustentaram empiricamente que o nível de conflito entre os pais, o nível de cooperação e o nível de triangulação seriam as dimensões centrais da coparentalidade. Por outro lado, Van Egeren et al. (2004) advogaram que a coparentalidade pode ser conceptualizada tanto por uma estrutura externa, em que consiste quem é que faz parte da díade coparental, como por uma estrutura interna, relativa às dimensões intraindividuais e intrafamiliares, exclusivas da aliança coparental. O modelo apresenta quatro dimensões de coparentalidade: solidariedade coparental, suporte coparental, parentalidade sabotada e parentalidade partilhada.

No entanto, a proposta teórica com maior evidência empírica é o Modelo da Estrutura Interna e Contexto Ecológico da Coparentalidade de Feinberg (2003) (Feinberg et al., 2016; Lamela, Figueiredo, Bastos \& Feinberg, 2016; Le, McDaniel, Leavitt \& Feinberg, 2016). Este modelo, que deriva da integração de propostas conceptuais anteriores e da evidência empírica produzida na literatura, apresenta os pressupostos de como a coparentalidade pode influenciar o ajustamento parental, a parentalidade e o ajustamento psicológico da criança. Adicionalmente, este modelo define diferentes dimensões da coparentalidade e teoriza como estas dimensões são influenciadas pelas características individuais de cada pai, pelos fatores familiares e pelo ambiente extrafamiliar (Feinberg, 2003; Feinberg et al., 2012). Nesta linha, Feinberg (2003) propôs que o subsistema coparental assenta em quatro dimensões: acordo nas práticas parentais, divisão do trabalho relacionado com a criança, suporte/sabotagem do papel coparental e gestão conjunta das relações familiares.

Mais detalhadamente, Feinberg (2003) descreve o acordo nas práticas parentais como a dimensão associada ao grau de entendimento entre a díade parental em assuntos relacionados com a criança, tais como os princípios morais, disciplina, formas de prestação de cuidados, decisões sobre a educação ou necessidades emocionais. A dimensão de divisão de tarefas, por sua vez, compreende a partilha entre os pais das obrigações das rotinas diárias de cuidados à criança, bem como da divisão das responsabilidades dos assuntos financeiros, médicos e legais relacionados com a criança. A dimensão suporte/sabotagem coparental consistente na qualidade e grau do suporte recíproco entre os membros da díade (e.g., expressões de afeto positivo, reforço, apoio emocional e respeito perante a autoridade do outro pai); o polo negativo desta dimensão (sabotagem) é manifestada por um padrão de hostilidade, crítica, culpa e afeto negativo perante o outro pai. Por fim, a dimensão gestão conjunta da família agrupa a gestão, pontuação e modelação das interações familiares, com especial foco nas interações entre os pais na prestação de cuidado às crianças.

Segundo este modelo, a qualidade das interações coparentais será o resultado da interdependência das dimensões, em que a variabilidade dos padrões de interação entre as famílias é consequência do tipo, grau e intensidade das ligações entre os componentes (Feinberg, 2003). Isto significa que o peso de cada dimensão da coparentalidade oscila de acordo com as características de cada díade coparental, de cada um dos pais e do seu contexto de desenvolvimento (Feinberg, 2003).

Do ponto de vista metodológico, foram desenvolvidos um conjunto de instrumentos de avaliação da coparentalidade, desde tarefas de observação coparental até medidas de autorrelato. Como medidas observacionais, o Coparenting and Family Rating (McHale, Kuersten-Hogan \& Lauretti, 2001) e o The Prenatal Lausanne Trilogue Play (Carneiro, Corboz-Warnery \& Fivaz-Depeursinge, 2006) apresentam tarefas padronizadas de interação diádica e triádica, aplicando um sistema de codificação de comportamentos coparentais 
específicos. Por outro lado, os instrumentos de autorrelato iniciais para avaliação da coparentalidade foram especialmente construídos para a sua medição em famílias com pais divorciados (e.g., Ahrons, 1981).

Posteriormente, foram desenvolvidos outros instrumentos direcionados a todos os pais independentemente do seu estado civil. Entre as medidas internacionalmente mais utilizadas para a avaliação da coparentalidade destacam-se a Parenting Alliance (PAM, Abidin \& Konold, 1999, versão portuguesa Lamela, Castro \& Figueiredo, 2013) e o Coparenting Questionnaire (CQ; Margolin et al., 2001, versão portuguesa Pedro \& Ribeiro, 2015). A PAM, que avalia a qualidade da relação entre os pais na prestação de cuidados aos filhos, demonstrou índices de validade de construto e de consistência interna, quer na sua versão original ( $\alpha=0.92)$, quer na versão portuguesa $(\alpha=0.95)$. Por sua vez, o Coparenting Questionnaire, que avalia a cooperação, a triangulação e o conflito coparentais, apresentou valores de validade de construto e de consistência interna, quer nas versões original e portuguesa ( $\alpha$ s entre 0.76 e 0.88 ).

Mais recentemente, Feinberg et al. (2012) desenvolveram a Coparenting Relationship Scale (versão portuguesa Escala da Relação Coparental). A ERC apresenta um conjunto de vantagens metodológicas e teóricas face às outras medidas de autorrelato. Em primeiro, a ECR foi concebida para avaliar as dimensões do modelo ecológico da coparentalidade (Feinberg, 2003), tornando-a na primeira medida que está baseada num modelo teórico da coparentalidade. Deste modelo, esta escala responde à principal crítica das medidas anteriores que, por serem consideradas ateóricas, não permitiam a compreensão e interpretação dos resultados obtidos (McHale, 1995; Schoppe-Sullivan, Mangelsdorf, Frosch \& McHale, 2004). Em segundo, a ERC apresentou estudos de validação que cumpriram os pressupostos recomendados para a validade psicométrica de instrumentos das medidas psicológicas (Feinberg et al., 2012).
No estudo original (Feinberg et al., 2012), a ERC foi validada através de um estudo longitudinal com uma amostra de 169 casais heterossexuais com bebés até os 2 anos de idade. Os autores realizaram um conjunto de procedimentos sequenciais para a validação da ECR. Em primeiro, selecionaram 47 itens de escalas anteriores de avaliação das relações interparentais e alocaram esses itens a sete subescalas definidas a priori para avaliarem as quatro dimensões da coparentalidade do modelo ecológico de Feinberg (2003). Assim, a dimensão acordo nas práticas parentais foi proposta ser avaliada por uma subescala com o mesmo nome. Por sua vez, a dimensão suporte/sabotagem foi ponderada ser avaliada por três subescalas: suporte coparental, sabotagem e aprovação parental. A dimensão divisão de tarefas foi proposta ser medida por uma subescala com a mesma denominação. A gestão conjunta da família foi definida a priori ser avaliada por uma subescala denominada por exposição ao conflito. Por fim, os autores da ECR conceberam uma última subescala, a proximidade coparental, que, apesar não medir uma dimensão de coparentalidade de Feinberg (2003), avalia o grau em que a relação coparental reforça a intimidade e a força da relação do casal.

A partir desta distribuição dos itens por subescalas definidas a priori, os autores conduziram uma análise fatorial exploratória e uma análise fatorial confirmatória que permitiu identificar itens que eram redundantes ou não se correlacionavam com os restantes itens da subescala que foram previamente alocados. Este procedimento reduziu o número de itens para 35 . De seguida, foi realizada uma análise fatorial confirmatória longitudinal para replicar e confirmar validade de construto da ECR. Após a confirmação da estrutura fatorial, os autores, por fim, testar a fiabilidade e a validade convergente e divergente da medida. No estudo de validação, os autores sugerem as qualidades psicométricas da ECR, demonstrando a validade de construto (com a confirmação estatística de uma solução fatorial com sete fatores), a consistência 
interna ( $\alpha=0.94$ para mães e $\alpha=0.91$ para pais) a validade convergente (e.g., correlações significativas, fortes e negativas com a uma medida de eficácia conjugal) e a validade divergente (e.g., correlações não significativas com uma medida de desejabilidade social) (Feinberg et al., 2012).

Segundo o nosso conhecimento, até à data, não existem estudos de validação psicométrica de outras versões internacionais desta escala publicados em revistas indexadas à PsycINFO, Scopus ou Web of Science (palavra-chave: Coparenting Relationship Scale; intervalo de publicação: janeiro de 2012-janeiro de 2018). No entanto, a ERC tem sido utilizada em estudos norte-americanos que avaliam, por exemplo, o apoio coparental na amamentação (Abbass-Dick, Stern, Nelson, Watson \& Dennis, 2015), estudos sobre os contributos da observação da coparentalidade e do temperamento da criança para o seu ajustamento sócio emocional (Altenburger, Lang, Schoppe-Sullivan, Kamp Dush \& Johnson, 2017), e também no desenvolvimento de uma medida de compreensão do gatekkeping maternal, em que se adaptou a ERC, para pais de crianças em idade pré-escolar, utilizando apenas cinco subescalas (Puhlman \& Pasley, 2017).

Versões em língua portuguesa com a ECR tem também sido utilizadas em investigações em Portugal e no Brasil. Globalmente, os resultados destes estudos revelaram associações teoricamente suportadas pelo modelo de Feinberg (2003) entre a coparentalidade medida pela ECR e outros construtos familiares, o que sugere a validade preditiva da escala. Por exemplo, Lamela et al. (2017) encontraram associações entre sintomas depressivos e somáticos maternos e o conflito coparental. Por sua vez, Böing e Crepaldi (2016), numa amostra de pais e mães brasileiros, mostraram que o suporte coparental se encontravam associado enquanto a sabotagem coparental se revelou relacionada com estilos parentais permissivos.

No entanto, nenhum destes estudos com amostras portuguesas ou brasileiras foram conduzidas com versões da ECR com propriedades psicométri- cas relatadas. A exceção é o estudo de Lamela et al. (2016) que demonstrou a validade de construto de uma versão reduzida da ECR numa amostra de pais divorciados portugueses. Neste estudo, pais divorciados que relataram valores mais elevados de conflito coparental apresentaram menores níveis de bem-estar subjetivo e relataram níveis mais elevados de sintomas de internalização e externalização nos filhos. Globalmente, estes resultados empíricos sugerem preliminarmente a adequação cultural da ECR para a população portuguesa.

O presente estudo teve com finalidade endereçar limitações existentes na literatura em Portugal sobre a validade de construto da ECR, sendo que ERC foi a primeira medida teoricamente orientada, cuja validade foi testada para a população portuguesa. $\mathrm{O}$ presente estudo pretendeu adicionar à literatura informação sobre como a presente escala se comportava na medição de coparentalidade em mães com filhos de diferentes faixas etárias, uma vez que os dados disponíveis da literatura internacional foram obtidos com amostras em pais com filhos até idade pré-escolar. Apesar da coparentalidade remeter para um construto diádico (pai-mãe) que se reflete nas interações triádicas dentro do sistema familiar (pai-mãe-filho), este construto pode ser avaliado apenas num dos membros da díade coparental, uma vez que as escalas de auto-relato da coparentalidade medem a perceção da mãe e/ ou do pai sobre os domínios da relação coparental (McHale, 2007).

Esta investigação teve como objetivo geral a adaptação e a validação da Escala de Relação Coparental de Feinberg et al. (2012) para a população portuguesa, utilizando uma amostra de mães portuguesas. O presente estudo teve quatro objetivos. Em primeiro, o estudo testou a validade de constructo da ECR. Hipotetizou-se que a estrutura fatorial da versão portuguesa apresentaria indicadores de validade de construto similares à solução fatorial da versão original. Em segundo, foi testada a fiabilidade da ECR, colocando-se a hipótese que o instrumento iria apresentar elevados 
valores de consistência interna. Em terceiro, testou-se a validade convergente da ECR. Partimos da hipótese que a ECR estaria positiva, forte e significativamente associada com os scores totais de uma medida de avaliação do acordo coparental e uma medida de satisfação conjugal. Finalmente, a presente investigação teve como objetivo examinar a validade divergente da ECR. Colocou-se em hipótese que a ECR ou estaria fracamente ou não estaria estatisticamente associada a uma medida de desejabilidade social.

\section{Método}

\section{Participantes}

A amostra foi constituída por 548 mães com uma relação conjugal $(71.2 \%$ eram casadas e $28.8 \%$ viviam com união de facto). A idade média foi de 39.5 anos $(D P=6.2$ anos $)$. A duração média da relação conjugal era de 13.29 anos $(D P=6.8$ anos). Aproximadamente, $33 \%$ das mães relataram terem uma escolaridade igual ou inferior ao ensino secundário e $67 \%$ das mães tinham um grau obtido no ensino superior. O rendimento financeiro médio era de 13.808 euros por ano ( $D P=764$ euros). No caso de a participante ter mais de um filho, foi pedido às mães que avaliassem a relação coparental com o seu parceiro, tendo como referência o filho mais novo ( $M$ número de filhos $=1.74, \mathrm{DP}=0.87)$. A idade média do filho-foco foi de 6.5 anos ( $D P=4.98$ anos; $50.6 \%$ rapazes).

\section{Medidas}

Escala de Relação Coparental -ERC(Feinberg et al., 2012). A Escala de Relação Coparental é uma medida de autorrelato composta, na sua versão original, por 35 itens. Os itens estão divididos em 7 subescalas: acordo nas práticas parentais (4 itens), suporte coparental (6 itens), sabotagem (6 itens), aprovação parental (7 itens), divisão de tarefas (2 itens), exposição ao conflito
(5 itens) e proximidade coparental (5 itens). Este questionário é classificado com uma escala tipo Likert, de 0 (não é verdadeiro sobre nós) a 6 (muito verdadeiro sobre nós), em que quanto maior for a pontuação, maior será a relação coparental. Os resultados de cada subescala são calculados pela média, existindo itens invertidos. Segundo os resultados americanos, os valores indicam a validade de construto, com uma forte consistência interna, em que os valores de Alpha de Cronbach variam entre 0.91 e 0.94 (Feinberg et al., 2012). Os itens da versão final portuguesa da ECR estão descritos na tabela 1 .

Tabela 1

Itens da Versão Final da ERC

Item

1. Acredito que o pai do meu filho é um bom pai.

2. A relação com o pai do meu filho é mais forte agora do que antes de termos um filho.

3. O pai do meu filho pergunta a minha opinião sobre assuntos relacionados com a sua educação.

4. O pai do meu filho presta grande atenção ao nosso filho.

5. É mais fácil e divertido brincar sozinha com o meu filho do que quando o pai está presente.

6. O pai do meu filho e eu temos ideias diferentes sobre como criar o nosso filho. (R)

7. O pai do meu filho diz-me que estou a fazer um bom trabalho ou faz-me saber de outra maneira que estou a ser uma boa mãe.

8. O pai do meu filho e eu temos ideias diferentes sobre rotinas de refeição, de sono e outras do nosso filho. (R)

9. Por vezes, o pai do meu filho faz piadas ou comentários sarcásticos sobre a minha forma de ser mãe.

10. O pai do meu filho não confia nas minhas capacidades enquanto mãe.

11. O pai do meu filho é sensível aos sentimentos e necessidades do nosso filho.

12. O pai do meu filho e eu temos diferentes critérios para o comportamento do nosso filho. (R)

13. O pai do meu filho tenta mostrar que é melhor do que eu a cuidar do nosso filho. 


\section{Item}

14. Sinto-me próxima do pai do meu filho quando o vejo a brincar com o nosso filho.

15. O pai do meu filho tem muita paciência com o nosso filho.

16. Às vezes conversamos sobre a melhor forma de dar resposta às necessidades do nosso filho.

17. Quando estamos juntos, o pai do meu filho às vezes compete comigo pela atenção do nosso filho.

18. O pai do meu filho mina a minha relação com o nosso filho.

19. O pai do meu filho está disposto a fazer sacrifícios pessoais para ajudar a cuidar do nosso filho.

20. Estamos a amadurecer e crescer juntos devido às nossas experiências enquanto pais.

21. O pai do meu filho aprecia o quanto me esforço para ser uma boa mãe.

22. Quando chego ao meu limite enquanto mãe, o pai do meu filho dá-me o apoio adicional que preciso.

23. O pai do meu filho faz-me sentir a melhor mãe possível para o nosso filho.

24. O pai do meu filho não gosta de ser incomodado por ele. (R)

25. Sermos pais tem-nos dado um sentido para o futuro.

26. Se sente no meio de uma interação tensa e sarcástica com o pai do seu filho?

\section{Discutem sobre o vosso filho à frente dele?}

28. Discutem sobre a vossa relação íntima ou outros assuntos não relacionados com o vosso filho à frente dele?

29. Um de vocês ou ambos dizem algo cruel ou ofensivo um ao outro à frente do vosso filho?

30. Gritam um com o outro a uma distância em que é possível que o vosso filho vos ouça?

${ }^{\mathrm{a}} \mathrm{O}$ número dos itens foi ajustado à solução fatorial portuguesa. A numeração apresentada não é equivalente à versão original, uma vez que foram eliminados 5 itens da versão portuguesa.

Nota. $(\mathrm{R})=$ Item revertido

Medida de Aliança Parental —PAM- (Abidin \& Konold, 1999; Versão Portuguesa: Lamela et al., 2013). Este é um instrumento que avalia a perceção que cada pai tem da tarefa coparental com o outro progenitor, medindo a qualidade da relação parental relativamente à prestação de cuidados da criança mais concretamente, a comunicação entre a díade parental sobre as práticas e estilos educativos, o compromisso mútuo quanto à educação e a compreensão das dificuldades e potencialidades parentais do outro pai (Lamela et al., 2013). A escala utilizada é a versão reduzida da PAM, composta por 6 itens, cotados por uma escala tipo Likert, variando entre 5 (concordo fortemente) e 1 (discordo fortemente), em que quanto mais altos forem os resultados apresentados, maior será a aliança parental entre os pais. Os valores psicométricos da versão portuguesa foram satisfatórios, com um valor da consistência interna elevado de 0.95 (Lamela et al., 2013). No presente estudo, o alfa de Cronbach foi de .89 .

Couples Satisfaction Index - CSI- (Funk \& Rogge, 2007) (Funk \& Rogge, 2007). A satisfação conjugal foi medida através da utilização da Escala de Índice de Satisfação do Casal-versão 4 itens (Funk \& Rogge, 2007). O CSI avalia a satisfação individual do parceiro da díade conjugal, numa escala de Likert de 7 pontos (entre 0 'discordo totalmente' até 6 'concordo totalmente'). Os resultados são obtidos através da soma da pontuação nos quatro itens, com um resultado intervalar de 4 a 24 pontos, em que pontuações mais elevadas predizem uma maior satisfação matrimonial. Este é um que instrumento apresenta excelentes propriedades psicométricas (e.g., alfa de Cronbach de (Funk \& Rogge, 2007). A versão portuguesa apresentou excelentes valores de consistência interna (alfa de Cronbach de 0.91) (Lamela et al., 2017). $\mathrm{Na}$ atual amostra, o alfa de Cronbach foi de 0.90 . Escala de Desejabilidade Social de Marlowe-Crowne - MCDS - (Ballard, 1992; Versão Portuguesa: Pechorro, Vieira, Poiares \& Marôco, 2012) (Ballard, 1992; Pechorro, Vieira, Poiares \& Marôco, 2012). A AMCDS é composta por 13 itens dicotómicos (verdadeiro e falso). Esta é uma escala que avalia a tendência de certas pessoas em apresentarem as suas qualidades de forma inflacionada ou exagerada, minimizando as suas 
fraquezas (Pechorro et al., 2012). A sua cotação é feita somando todos os itens, após a reversão dos itens sinalizados. A versão portuguesa demonstrou características psicométricas satisfatórias (coeficiente de Kuder-Richardson de 0.60 para consistência interna) (Pechorro et al., 2012). Na atual amostra, a consistência interna foi de 0.72 (coeficiente de Kuder-Richardson).

\section{Procedimentos}

Foi conduzido um estudo online desenhado especificamente para recolha de dados para examinar os objetivos da presente investigação. Estabeleceram-se quatro critérios de inclusão para a participação no estudo: ser do sexo feminino, ter idade igual ou superior a 18 anos, ter uma relação íntima heterossexual e pelo menos um filho com o atual parceiro conjugal. O questionário foi hospedado num servidor seguro de uma universidade portuguesa durante quatro meses. $\mathrm{Na}$ primeira parte do inquérito, foi apresentado um consentimento informado onde se expuseram os objetivos, os critérios de inclusão, procedimentos do estudo, procedimentos da confidencialidade, procedimentos de consentimento ou não consentimento de participação, métodos de tratamento dos dados e os contactos da equipa de investigação. Foi garantido às participantes o total anonimato das respostas, dado que não foram questionadas informações que diretamente podiam identificar a participante (e.g., nome ou email), nem foram recolhidas informações sobre o endereço IP nem outro dado de identidade informática. O consentimento informado era obtido ao selecionar a opção de 'página seguinte', que correspondia ao acesso ao inquérito, cujo o tempo estimado de preenchimento era de 20 a 25 minutos. Não foi fornecida qualquer compensação monetária. A divulgação do estudo foi realizada através de emails das instituições do ensino superior, fóruns online sobre família e nas redes sociais. A investigação foi realizada de acordo com o Código de Deontologia da Ordem Psicólogos Portugueses e com a Declaração de Helsínquia. Foram também seguidas as recomendações metodológicas e éticas para a investigação online da American Psychological Association, tais como, por exemplo, a aplicação de procedimentos de consentimento informado e o desenho de um plano parcimonioso de recrutamento de participantes (Kraut et al., 2014).

Numa fase prévia às análises estatísticas, foram aplicados procedimentos de limpeza da base de dados recomendados por Funk e Rogge (2007). Assim, foram eliminadas participantes que: 1) não cumpriram os critérios de inclusão, (2) afirmaram não ser a primeira a vez que estavam a responder ao protocolo; (3) não completaram $70 \%$ do total do protocolo e/ou deixaram quatro itens por preencher do instrumento que avaliava a variável dependente. Das 612 participações contabilizadas pelo sistema informático de recolha de dados, foram invalidadas 64 participações $(10.5 \%$ do total de participantes) por não cumprirem pelo menos um destes três critérios. Após estes procedimentos de limpeza de dados, a amostra final foi composta por 548 participantes. Não foram encontradas diferenças estatisticamente significativas nas variáveis demográficas entre as participantes que permaneceram na amostra final e as participantes que foram eliminadas, nomeadamente na idade, $t(610)=0.76, n s .$, nos anos de relacionamento, $t(610)=-0.12, n s$. , e no número de filhos $t(366)$ $=-0.76, \mathrm{~ns}$.

\section{Procedimentos estatísticos}

Para testar a validade de construto da versão portuguesa da ECR, foi conduzida uma análise fatorial confirmatória - $\mathrm{AFC}$ - , recorrendo ao Amos 20. Foi utilizado o método de estimação de máxima verossimilhança (Kline, 2015). Foram utilizados quatro índices para examinar a adequação dos modelos testados: o Comparative Fit index 
- CFI-, o Normed Fit Index - NFI-, o Tucker-Lewis Index - TLI- e o Root-Mean-Square Error of Approximation — RMSEA- Valores superiores a 0.90 nos índices CFI, NFI e TLI e um valor RMSEA igual ou inferior a 0.05 indicam um bom ajustamento (Bollen \& Long, 1993; Kline, 2015). No objetivo dois, para investigar a fiabilidade da ECR, foi conduzida uma análise de consistência de alfa de Conbrach. Utilizaram-se as recomendações de Nunnally e Bernstein (1994) para análise dos alfas de Cronbach, em que valores iguais ou superiores a 0.70 indicam bons níveis de fiabilidade. $\mathrm{O}$ terceiro e quarto objetivos foram testados através de correlações bivariadas de Pearson entre a ERC e os instrumentos utilizados como medidas de referência de validade convergente (PAM e CSI) e divergente (MSCD-SF). As análises estatísticas dos objetivos dois, três e quatro foram conduzidas recorrendo ao IBM SPSS Statistics 23.

\section{Resultados}

\section{Validade de construto}

Foi conduzida uma AFC para testar a estrutura fatorial da versão portuguesa da ERC, recorrendo ao Amos 20. O modelo original de 7 fatores foi testado. Para o total da amostra, o modelo testado não permitiu nenhuma correlação entre termos de erro. Este modelo mostrou um ajustamento pobre, $\chi^{2}(539)=1721.6, n s, \mathrm{CFI}=0.88, \mathrm{NFI}=0.84$ e RMSEA $=0.063(90 \%$ IC $=0.06-0.07)$. Analisando os valores de saturação dos itens por fator, assim como a intercorrelação entre fatores, verificou-se que o fator 'Divisão de Tarefas' não apresentava qualquer intercorrelação significativa com os demais fatores $(<0.20, n s)$. Adicionalmente, os itens que compunham este fator (itens 5 e 20), não saturavam significativamente no fator $(0.21$ e 0.14 , respetivamente). Analisando as saturações dos outros itens nos fatores originais, verificou-se que três itens não saturavam significativamente nos fatores originais: o item 6 do fator 'Acordo' (-0.06), o item 7 do fator 'Aprovação' (0.12) e o item 28 do fator 'Proximidade' (0.17). Com base em fundamentação teórica e as recomendações metodológicas de Kline (2015), os itens do fator 'Divisão de Tarefas' e os itens 6, 7 e 28 foram eliminados.

Após esta remoção, a nova estrutura fatorial de 6 fatores foi testada. Os valores de adequação do modelo melhoraram significativamente em comparação com o primeiro modelo testado, $\chi^{2}(382)$ $=1061.29, n s, \mathrm{CFI}=0.91, \mathrm{NFI}=0.88$ e RMSEA $=$ $0.058(90 \% \mathrm{IC}=0.057-0.053)$. Apesar de estarem próximos dos valores recomendados para verificação da adequação da estrutura fatorial, o presente modelo não apresentou indicadores satisfatórios de ajustamento. Analisando os índices de modificação do modelo, verificou-se que o modelo requeria 8 correlações de dois termos de erro com vista a atingir a sua adequação (variação dos índices de modificação entre 12.432 e 43.455). Considerando que as correlações entre termos de erro de itens que saturavam em dois fatores ('Suporte' e 'Aprovação') que avaliam conceptualmente a dimensão 'Suporte coparental' do modelo de Feinberg (2003), decidiu-se que estas correlações são teoricamente coerentes e defensáveis, o que possibilita a sua inclusão no modelo (Cole, Ciesla \& Steiger, 2007; Kline, 2015). Assim, as correlações dos termos de erro foram especificadas ao mesmo tempo no modelo (Kline, 2015). Testou-se, de seguida, novamente este modelo de 6 fatores, permitindo a correlação entre os 8 termos de erros, que foram incluídos no mesmo momento. Esta solução atingiu a adequação, $\chi^{2}(372)=828.5, n s, \mathrm{CFI}=0.95$, NFI $=0.91$ e RMSEA $=0.04(90 \% \mathrm{IC}=0.04-0.05)$. As saturações dos itens por fatores, as covarâncias entre fatores e as correlações de termos de erro estão apresentadas na figura 1. As análises estatísticas subsequentes para testar a fiabilidade e validade convergente e divergente foram realizadas com base nesta distribuição de itens pelos 6 fatores. 


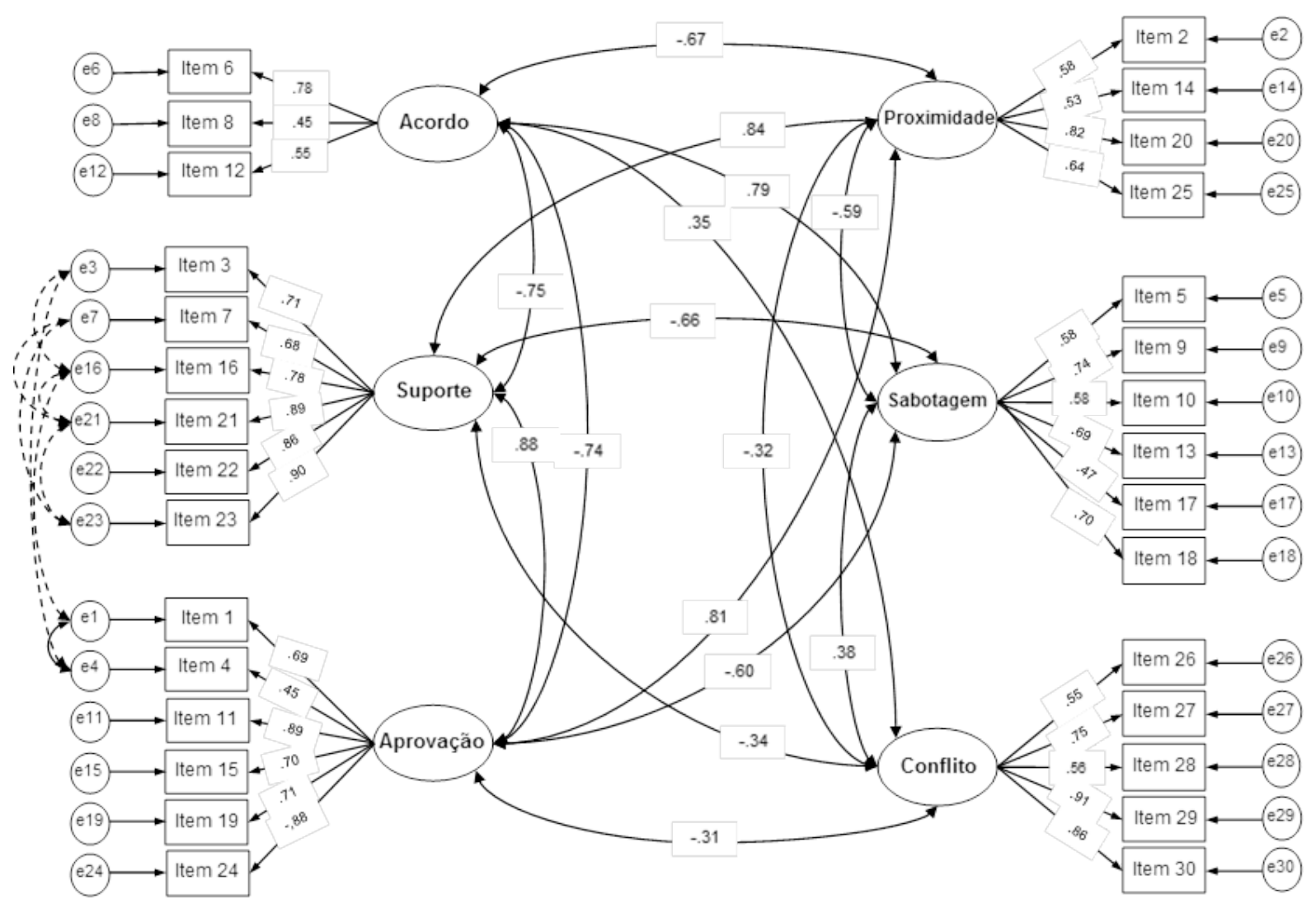

Figura 1. Modelo confirmatório de seis fatores da versão portuguesa da ECR. Setas curvadas em linha contínua expressam as correlações entre os fatores da ECR. Setas curvadas em linha tracejada refletem as correlações entre termos de erro. Todas as correlações entre os fatores da ECR foram significativas a 0.001 . São também indicadas as saturações item-fator

\section{Consistência interna e Validades Convergente e Divergente da ERC}

Consistência interna. A consistência interna das subescalas da ERC foi examinada através da análise dos alfas de Cronbach. Na tabela 2, estão descritos os valores de alfa de Cronbach, a correlação média entre itens e a variação (mínimo-máximo) das correlações item-total para cada das subescalas e total da ERC. Tendo em consideração as recomendações para análise dos alfa de Cronbach (Nunnally \& Bernstein, 1994), em que valores iguais ou superiores a 0.70 indicam bons níveis de fiabilidade, todas as subescalas apresentaram bons ou excelentes de fiabilidade.
Validade convergente e validade divergente. Correlações entre o total da ERC e as medidas utilizadas para testar a validade convergente e divergente estão descritas na tabela 3. Foi encontrada uma correlação elevada, positiva e significativa entre o total da ERC e a PAM-R e uma correlação moderada, positiva e significativa entre a ERC e o CSI. Por outro lado, não foi encontrada uma associação significativa entre a ERC e a MCSDS-SF.

\section{Discussão}

O objetivo deste estudo foi avaliar as propriedades psicométricas da versão portuguesa da ERC numa amostra portuguesa de mães numa relação íntima (casamento ou em união de facto). A ERC 
Tabela 2

Valores de Consistência Interna das Subescalas da ERC

\begin{tabular}{lcccccccc}
\hline & \multirow{2}{*}{ Total ERC } & \multicolumn{5}{c}{ Subescalas } \\
\cline { 3 - 8 } & & Acordo & Proximidade & Suporte & Aprovação & Sabotagem & Conflito \\
\hline Alfa Cronbach & 0.74 & 0.70 & 0.84 & 0.94 & 0.77 & 0.79 & 0.81 \\
Correlação em itens & 0.07 & 0.44 & 0.57 & 0.75 & 0.32 & 0.44 & 0.49 \\
Variação correlações item-total & $-0.06-0.69$ & $0.46-0.56$ & $0.63-0.76$ & $0.76-0.89$ & $-0.47-0.83$ & $0.50-0.69$ & $0.43-0.76$ \\
\hline
\end{tabular}

Tabela 3

Validades Convergente e Divergente

\begin{tabular}{lccc}
\hline & 1. ERC & 2. PAM-R & 3. CSI \\
\hline 1. ERC & - & & \\
2. PAM-R & $0.65^{*}$ & & \\
3. CSI & $0.32^{*}$ & $0.53^{*}$ & $-0.17^{*}$ \\
4. MCSDS-SF & 0.06 & -0.06 & \\
\hline
\end{tabular}

Nota . ERC = Escala de Relação Coparental; PAM-R = Medida da Aliança Parental-Revista; CSI $=$ Couple Satisfaction Index; MCSDS-SF = Escala de Desejabilidade Social de Marlowe-Crowne. $* \mathrm{p}<0.001$

foi desenhada para avaliar empiricamente as quatro dimensões da coparentalidade propostas por Feinberg (2003), subdividida em 7 fatores. Os resultados obtidos da análise fatorial confirmatória mostraram o não-ajustamento do modelo original de 7 fatores aos dados. Com a análise dos valores de saturação, bem como com a intercorrelação entre os fatores, foi possível observar que o fator "Divisão de Tarefas" não apresentava uma intercorrelação significativa com os restantes fatores, assim como os itens que compunham esse mesmo fator (5 e 20), o que conduziu à sua eliminação (Kline, 2015). Após esta remoção, a estrutura fatorial de 6 fatores com existência de correlação de termos de erro foi testada, revelando uma melhoria significativa, em comparação com o primeiro modelo testado de 7 fatores.

Apesar da inclusão do fator 'Divisão de Tarefas' na estrutura fatorial da versão original da ECR (Feinberg et al., 2012), os resultados obtidos nesse estudo relativos a este fator demonstraram uma intercorrelação mais baixa (0.46), comparativamente com os restantes fatores, revelando que, no que concerne à divisão de tarefas, a consistência interna é mais fraca nos pais, em relação as mães (Feinberg et al., 2012). Para Feinberg et al. (2012) é possível que a dimensão 'Divisão de Tarefas' seja menos coesiva, recomendando assim um maior foco nas investigações futuras sobre este fator específico.

Este resultado pode, por isso, refletir-se como um contributo na discussão conceptual sobre as dimensões nuclear que compõem o construto da coparentalidade. A não-saturação dos itens do fator 'divisão de tarefas' da versão original da ECR corroboram as preposições de alguns investigadores que advogam que a natureza, frequência e reciprocidade da divisão de tarefas no cuidado das crianças não contribuem para a definição conceptual da coparentalidade (e.g. McHale, 2007; Lamela et al., 2016). Por exemplo, McHale (2007) enfatiza que a coparentalidade não deve primeiramente focar-se sobre a forma como os adultos partilham a divisão de trabalho nos cuidados às crianças, uma vez que esta divisão é culturalmente situada, existindo acentuadas diferenças em função da cultura e da 
organização social das várias sociedades. Nesse sentido, a incorporação da divisão do trabalho coparental como dimensão basilar na operacionalização da coparentalidade implicaria um viés etnocêntrico, favorecendo as conceções ocidentais sobre o funcionamento e organização familiares. Consequentemente, rejeitando o pressuposto que é a divisão do trabalho na prestação de cuidados à criança o núcleo deste construto, McHale (2007) preconiza que a coparentalidade define o grau de colaboração, afirmação e suporte entre os adultos no cuidado e educação das crianças por quem partilham a responsabilidade. Assim, o presente estudo parece corroborar resultados de investigação anterior que sugerem que a divisão de tarefas como um produto da qualidade da relação coparental, sendo, dessa forma, um construto distinto e não integrante da coparentalidade (McHale, Kuersten-Hogan \& Rao, 2004).

Quanto aos valores de consistência interna, os resultados foram reveladores de um coeficiente igual ou superior a 0.70 , apresentando assim uma boa fiabilidade (Nunnally \& Bernstein, 1994). No estudo original da escala, os resultados apresentaram semelhanças com os obtidos neste estudo de validação, com um alfa de Cronbach entre 0.75 0.90. Valores de consistência interna similares foram encontrados em estudos portugueses com versões reduzidas da ECR (e.g., Lamela et al., 2016).

Por fim, a validade convergente e divergente foi demonstrada. Ao nível da convergência convergente, foi encontrada uma correlação positivamente elevada entre o total da ERC e a PAM-R. Estes resultados revelaram a existência de uma associação positiva entre o acordo parental na relação coparental (medido pela PAM-R) com as dimensões da coparentalidade. Esta elevada correlação era esperada, uma vez que ambas escalas estão a medir construtos similares. Note-se, no entanto, que a PAM-R mede o acordo coparental, o que, de acordo com o modelo ecológico de Feinberg (2003), é apenas uma das dimensões da coparentalidade. Adicionalmente, uma correlação moderada, positiva e significativa foi encontrada, entre a ERC e CSI. Os resultados do presente estudo estão linha com os descritos no estudo original de Feinberg et al. (2012), em que foram observadas correlações, de moderadas a fortes, entre o papel coparental e a relação conjugal. Tal como esperado, esta correlação não foi elevada, uma vez apesar de teoricamente ser esperado que satisfação na relação conjugal prediga a coparentalidade, elas não traduzem os mesmos construtos e refletem diferentes processos familiares (Feinberg, 2003). Este resultado corrobora o pressuposto concetual da autonomia do subsistema coparental face ao subsistema conjugal (Feinberg, 2003; Margolin et al., 2001; McHale, 1995). Assim, caso a relação coparental fosse um substrato da relação conjugal, estas duas componentes do sistema familiar estariam sempre altamente correlacionadas, o que não se verificou nos estudos realizados com a ECR e na presente investigação.

Por fim, na avaliação da validade divergente, não foi encontrada uma associação entre a ERC e a MCDS-SF. Estes valores obtidos estão em linha aos relatados no estudo de validação da versão original da ERC (Feinberg et al., 2012). Estes resultados sugerem que a desejabilidade social é um construto diferente da coparentalidade, uma vez que a avaliação da relação coparental não se mostrou associada com a tendência das participantes em apresentarem a sua relação coparental da forma mais favorável relativa às normas sociais explícitas e/ou implícitas.

\section{Limitações e conclusões}

Este estudo apresenta algumas limitações que devem ser consideradas na interpretação dos resultados. Primeiramente, foi utlizada uma amostra por conveniência, através de um questionário online, $\mathrm{o}$ que poderá diminuir a possibilidade de generalização dos resultados. A amostra foi constituída por participantes com níveis elevados de educação. $\mathrm{O}$ elevado nível de educação das participantes poderá 
estar associado ao fato do tipo de divulgação do estudo. Em segundo lugar, não foram incluídos pais e mães/pais divorciados ou separados. Apesar neste estudo online ter sido respondido por alguns participantes homens e mulheres divorciadas/ separadas, foi decidido metodologicamente não usar estes dados, uma vez que a distribuição dos participantes por género e estado civil não representava a população e poderia criar enviesamento estatístico. Esta homogeneidade entre os participantes permitiu aumentar o poder estatístico das análises. No entanto, esta variabilidade limitada diminui a possibilidade de generalização destes resultados a pais e a mães com outro estatuto civil e a díades coparentais compostas por pais do mesmo sexo. Considerando as taxas de divórcio em Portugal e as potenciais diferenças entre género na avaliação da coparentalidade, estudos futuros devem aumentar a variabilidade sóciodemográfica entre os participantes, a fim de confirmar a validade da ERC, recorrendo, por exemplo, a análises confirmatórias multigrupo. Em particular, sugere-se o prosseguimento dos estudos de validação em amostras de pais divorciados, uma vez que a qualidade da coparentalidade tem sido significativamente associada ao funcionamento psicológico dos filhos após a dissolução conjugal dos pais (Lamela \& Figueiredo, 2016; Lamela et al., 2016) (e.g., Lamela \& Figueiredo, 2016; Lamela et al., 2016). Adicionalmente, investigação futura sobre a escala deverá também analisar a relação entre a avaliação da coparentalidade das mães e dos pais na mesma díade coparental, assim como testar as suas associações com outros construtos psicológicos, recorrendo a instrumentos atualmente validados para a população portuguesa.

Apesar das limitações existentes, o presente estudo demonstra preliminarmente que a versão portuguesa da ERC cumpre os indicadores de qualidade psicométrica e pode ser utilizada em contexto de investigação. Aconselha-se a uma utilização cautelosa da ERC em contexto clínico, até novos estudos confirmarem a sua estrutura fatorial. Em
Portugal, do nosso conhecimento, só duas escalas estão validadas para medição da coparentalidade (Coparenting Questionnaire e a Medida de Aliança Parental). No entanto, estas escalas foram construídas sem ter um modelo teórico subjacente e a seleção dos itens não obedeceu a qualquer mapa teórico, mas sim a decisões de eficiência estatística. Este estudo, ao apresentar os resultados da validação de uma escala construída para medir as dimensões da coparentalidade defendidas por um modelo teórico de referência no domínio, contribui para responder a estas limitações metodológicas da investigação anterior.

\section{Referências}

Abbass-Dick, J., Stern, S., Nelson, L., Watson, W. \& Dennis, C.-L. (2015). Coparenting breastfeeding support and exclusive breastfeeding: A randomized controlled trial. Pediatrics, 135(1), 102-110. DOI: https://doi.org/10.1542/ peds.2014-1416

Abidin, R. \& Konold, T. (1999). Parenting Alliance Measure: Professional manual. Odessa: Psychological Assessment Resources.

Ahrons, C. R. (1981). The continuing coparental relationship between divorced spouses. American Journal of Orthopsychiatry, 51(3), 415-428. DOI: https://doi.org/10.1111/j.1939-0025.1981. tb01390.x

Altenburger, L., Lang, S., Schoppe-Sullivan, S., Kamp Dush, C. \& Johnson, S. (2017). Toddlers' differential susceptibility to the effects of coparenting on social-emotional adjustment. International Journal of Behavioral Development, 41(2), 228-237. DOI: https://doi. org/10.1177/0165025415620058

Ballard, R. (1992). Short forms of the Marlow-Crowne Social Desirability Scale. Psychological Reports, 71(3f), 1155-1160. DOI: https://doi. org/10.2466/pr0.1992.71.3f.1155

Belsky, J., Crnic, K. \& Gable, S. (1995). The determinants of coparenting in families with toddler 
boys: Spousal differences and daily hassles. Child Development, 66(3), 629-642. DoI: https:// doi.org/10.1111/j.1467-8624.1995.tb00894.x

Böing, E. \& Crepaldi, M. A. (2016). Relação pais e filhos: Compreendendo o interjogo das relações parentais e coparentais. Educar Em Revista, 59, 17-33. DOI: https://doi.org/10.1590/01044060.44615

Bollen, K. \& Long, J. (1993). Testing structural equation models. Newbury Park: Sage Publications.

Bronte-Tinkew, J., Horowitz, A. \& Carrano, J. (2010). Aggravation and stress in parenting: Associations with coparenting and father engagement among resident fathers. Journal of Family Issues, 31(4), 525-555. DOI: https://doi. org/10.1177/0192513X09340147

Carneiro, C., Corboz-Warnery, A. \& Fivaz-Depeursinge, E. (2006). The Prenatal Lausanne Trilogue Play: A new observational assessment tool of the prenatal co-parenting alliance. Infant Mental Health Journal, 27(2), 207-228. DOI: https://doi.org/10.1002/imhj.20089

Cole, D. A., Ciesla, J. A. \& Steiger, J. H. (2007). The insidious effects of failing to include design-driven correlated residuals in latent-variable covariance structure analysis. Psychological Methods, 12(4), 381-398. DoI: https://doi.org/10.1037/1082-989X.12.4.381

Delvecchio, E., Sciandra, A., Finos, L., Mazzeschi, C. \& Riso, D. Di. (2015). The role of co-parenting alliance as a mediator between trait anxiety, family system maladjustment, and parenting stress in a sample of non-clinical Italian parents. Frontiers in Psychology, 6, 1177. DoI: https:// doi.org/10.3389/fpsyg.2015.01177

Van Egeren, L. A. \& Hawkins, D. P. (2004). Coming to terms with coparenting: Implications of definition and measurement. Journal of Adult Development, 11(3), 165-178. DoI: https://doi. org/10.1023/B:JADE.0000035625.74672.0b

Fagan, J., Levine, E. C., Kaufman, R. \& Hammar, C. (2016). Low-income, nonresident fathers' coparenting with multiple mothers and rela- tives: Effects on fathering. Journal of Family Psychology, 30(6), 665-675. DOI: https://doi. org/10.1037/fam0000231

Favez, N., Tissot, H. \& Frascarolo, F. (2016). Parents' representations of mother-child and father-child relationships as predictors of early coparenting interactions. Journal of Family Studies, 1-15. DOI: https://doi.org/10.1080/13 229400.2016.1230511

Feinberg, M. E. (2003). The internal structure and ecological context of coparenting: A framework for reserch and intervention. Parenting: Science and Practice, 3(2), 95-131. DoI: https://doi. org/10.1207/S15327922PAR0302

Feinberg, M. E., Brown, L. D. \& Kan, M. L. (2012). A multi-domain self-report measure of coparenting. Parenting, Science and Practice, 12(1), 1-21. DOI: https://doi.org/10.1080/15295192.2 012.638870

Feinberg, M. E., Jones, D. E., Roettger, M. E., Hostetler, M. L., Sakuma, K.-L., Paul, I. M. \& Ehrenthal, D. B. (2016). preventive effects on birth outcomes: Buffering impact of maternal stress, depression, and anxiety. Maternal and Child Health Journal, 20(1), 56-65. DoI: https:// doi.org/10.1007/s10995-015-1801-3

Funk, J. L. \& Rogge, R. D. (2007). Testing the ruler with item response theory: Increasing precision of measurement for relationship satisfaction with the Couples Satisfaction Index. Journal of Family Psychology, 21(4), 572-583. DOI: https://doi.org/10.1037/0893-3200.21.4.572

Kline, R. (2015). Principles and practice of structural equation modeling. New York: Guilford.

Korja, R., Piha, J., Otava, R., Lavanchy-scaiola, C., Ahlqvist-Björkroth, S., Aromaa, M. \& Räihä, H. (2016). Mother's marital satisfaction associated with the quality of mother-father-child triadic interaction. Scandinavian Journal of Psychology, 57(4), 305-312. DOI: https://doi. org/10.1111/sjop.12294

Kuersten-Hogan, R. (2017). Bridging the gap across the transition to coparenthood: triadic inte- 
ractions and coparenting representations from pregnancy through 12 months postpartum. Frontiers in Psychology, 8, 475. DOI: https:// doi.org/10.3389/fpsyg.2017.00475

Lamela, D., Castro, M. \& Figueiredo, B. (2013). Medida da aliança parental: Validação portuguesa e construção de uma versão reduzida. Archives of Clinical Psychiatry (São Paulo), 40(6), 215-219. DOI: https://doi.org/10.1590/ S0101-60832013000600002

Lamela, D. \& Figueiredo, B. (2016). Coparenting after marital dissolution and children's mental health: A systematic review. Jornal de Pediatria, 92(4), 331-342. DOI: https://doi.org/10.1016/j. jped.2015.09.011

Lamela, D., Figueiredo, B., Bastos, A. \& Feinberg, M. (2016). Typologies of post-divorce coparenting and parental well-being, parenting quality and children's psychological adjustment. Child Psychiatry and Human Development, 47(5), 716-728. DOI: https://doi.org/10.1007/s10578015-0604-5

Lamela, D., Jongenelen, I., Morais, A. \& Figueiredo, B. (2017). Cognitive-affective depression and somatic symptoms clusters are differentially associated with maternal parenting and coparenting. Journal of Affective Disorders, 219, 37-48. DOI: https://doi.org/10.1016/j.jad.2017.05.006

Lamela, D., Nunes-Costa, R. \& Figueiredo, B. (2010). Modelos teóricos das relações coparentais: Revisão crítica. Psicologia Em Estudo, 15(1), 2015-216. DOI: https://doi.org/10.1590/S141373722010000100022

Le, Y., McDaniel, B. T., Leavitt, C. E. \& Feinberg, M. E. (2016). Longitudinal associations between relationship quality and coparenting across the transition to parenthood: A dyadic perspective. Journal of Family Psychology, 30(8), 918-926. DOI: https://doi.org/10.1037/fam0000217

Margolin, G., Gordis, E. B. \& John, R. S. (2001). Coparenting: A link between marital conflict and parenting in two-parent families. Journal of Family Psychology, 15(1), 3-21. DOI: https:// doi.org/10.1037/0893-3200.15.1.3

McHale, J. (2007). When infants grow up in multiperson relationship systems. Infant Mental Health Journal, 28(4), 370-392. DOI: https:// doi.org/10.1002/imhj.20142

McHale, J. (1995). Coparenting and triadic interactions during infancy: The roles of marital distress and child gender. Developmental Psychology, 31(6), 985-996. DOI: https://doi. org/10.1037/0012-1649.31.6.985

McHale, J. (1997). Overt and covert coparenting processes in the family. Family Process, 36(2), 183-201. Retrieved from http://www.ncbi.nlm. nih.gov/pubmed/9248827

McHale, J., Kuersten-Hogan, R. \& Lauretti, A. (2001). Evaluating coparenting and family-level dynamics during infancy and early childhood: The coparenting and family rating scale. Em P. Kerig \& K.-M. Lindahl (eds.), Family observational coding systems. Resources for systemic research (pp. 151-170). Mahwah: Laurence Erlbaum Associates.

McHale, J., Kuersten-Hogan, R. \& Rao, N. (2004). Growing points for coparenting theory and research. Journal of Adult Development, 11(3), 221-234. DOI: https://doi.org/10.1023/B:JADE.0000035629.29960.ed

McHale, J. \& Lindahl, K.-M. (2011). Coparenting: A conceptual and clinical examination of family systems. Washington DC: American Psychological Association.

Murphy, S. E., Jacobvitz, D. B. \& Hazen, N. L. (2016). What's so bad about competitive coparenting? Family-level predictors of children's externalizing symptoms. Journal of Child and Family Studies, 25(5), 1684-1690. DOI: https:// doi.org/10.1007/s10826-015-0321-5

Nunnally, J. \& Bernstein, I. (1994). Psychometric Theory. New York: McGraw-Hill.

Pechorro, P., Vieira, R., Poiares, C. \& Marôco, J. (2012). Contributos para a validação duma 
versão vurta da Escala de Desejabilidade Social de Marlowe-Crowne com adolescentes portugueses. Arquivos de Medicina, 26(3), 103-108.

Pedro, M. \& Ribeiro, T. (2015). Adaptação portuguesa do Questionário de Coparentalidade: Análise fatorial confirmatória e estudos de validade e fiabilidade. Psicologia: Reflexão e Crítica, 28(1), 116-125. DOI: https://doi.org/10.1590/16787153.201528113

Pedro, M., Ribeiro, T. \& Shelton, K. H. (2012). Marital satisfaction and partners' parenting practices: The mediating role of coparenting behavior. Journal of Family Psychology, 26(4), 509-522. DOI: https://doi.org/10.1037/a0029121 Puhlman, D. J. \& Pasley, K. (2017). The Maternal Gatekeeping Scale: Constructing a measure. Family Relations, Advance online publication. DOI: https://doi.org/10.1111/fare.12287

Schoppe-Sullivan, S. J., Mangelsdorf, S. C., Frosch, C. A. \& McHale, J. L. (2004). Associations between coparenting and marital behavior from infancy to the preschool years. Journal of Family Psychology, 18(1), 194-207. Dor: https:// doi.org/10.1037/0893-3200.18.1.194
Recebido: abril 14, 2017

Aprovado: fevereiro 2, 2018 